\title{
Institucionalismo y nuevos enfoques políticos
}

Manuel Menéndez-Alzamora

Recibido: 19/09/2014 - Aceptado: 15/12/2014

\section{Resumen}

Después del dominio en la Ciencia Política de las corrientes conductistas se produce, desde finales de los años setenta y principios de los noventa, una recuperación del institucionalismo, lo que ha sido denominado nuevo institucionalismo, algo que de manera simbólica se conoce como la reincorporación del Estado (Almond, 1985). Esta reincorporación del Estado es un fenómeno ciertamente intenso en los Estados Unido s en la medida que el Estado nunca desapareció del todo, en la tradición europea, de la Ciencia Política (Peters, 2003); particularmente esto es cierto en la tradición española. Este artículo aborda el tema desde la historia y sus principales postulados, haciendo una reflexión al respecto de la institucionalidad del Estado y los nuevos enfoques políticos.

\section{Palabras clave}

Institucionalidad, enfoques políticos, procesos políticos integradores.

\begin{abstract}
A recovery of institutionalism was produced after the dominance of behaviorist approaches in the Political Sciences from the late seventies to the beginning of the nineties; this has been called "new institutionalism." It is something that has somehow been symbolically called the "reincorporation of the State" (Almond, 1985). This State reincorporation is a rather intense phenomenon in the United States in the way that the State never disappeared in its entirety in the european tradition of the Political Sciences. (Peters, 2003); this is particularly true in the Spanish tradition. This article addresses the topic from a historical point of view and its main postulates, reflecting on the institutionality of the State and the new political approaches.
\end{abstract}

\section{Keywords}

Institutionality, political approaches, integrating political processes.

\footnotetext{
Manuel Menéndez Alzamora es Doctor en Derecho por la Universitat de València. Es autor del libro La generación del 14 (Madrid, Siglo XXI, 2006), que obtuvo el Premio Nacional de la Asociación Española de Ciencia Política (Aecpa) al mejor libro editado en 2006 y el XI Premio Nacional Ángel Herrera 2007 al mejor trabajo de investigación. Cuenta con múltiple trabajo intelectual en publicaciones de libros y artículos especializados, capítulos en obras colectivas y trabajos en actas de congresos nacionales e internacionales. Su labor docente ha estado ligada a la Ciencia Política en los ámbitos de la Teoría del Estado, Teoría Política y el Pensamiento Político, así como a las Libertades Públicas, los Derechos Fundamentales y las Instituciones Políticas como profesor de "Teoría Política", "Fundamentos de Ciencia Política", "Pensamiento Político Contemporáneo", "Libertades Públicas" y "Derecho de la información", entre otras asignaturas impartidas a lo largo de 23 cursos académicos completos con dedicación plena y exclusiva. Profesor del Área de Ciencia Política y de la Administración. Director académico adjunto en el Máster en Liderazgo Político y Dirección de Instituciones político-administrativas (on line) de la Universidad de Alicante, España.
} 


\section{Institucionalismo y Ciencia Política}

El movimiento del nuevo institucionalismo toma nombre y se inicia en el texto de James March y Johan Olsen, "The New Institutionalism: Organizational Factors in Political Life", de 1984 (March y Olsen, 2003). Como señalan David Marsh y Heather Savigny, este nuevo institucionalismo significa muchas cosas (March y Savigny, 2004). Hall y Taylor proponen tres formas principales de nuevo institucionalismo: elección racional, sociológico e histórico (Hall y Taylor, 1996). Por su parte, Peters plantea una tipología con siete variantes: normativo, elección racional, histórico, empírico, sociológico, instituciones de representación de intereses e institucionalismo internacional (Peters, 2003). Por último, Lowndes propone tres corrientes en el institucionalismo: internacional, histórico y empírico que presenta como contrapeso a la clásica diferenciación entre la elección racional y la aproximación normativa (Lowndes, 2002).

De entre todas estas propuestas vamos a decantarnos por el esquema de Guy Peters, que plasma este nuevo institucionalismo en seis direcciones que son: institucionalismo normativo, institucionalismo de la elección racional —Racional Choice Institutionalism-, institucionalismo histórico - Historical Institutionalism-, institucionalismo empírico - Empirical Institutionalism-, institucionalismo social - Social Institutionalisme institucionalismo internacional -(International Institutionalism) - . Consideramos que las cuatro primeras configuran el núcleo duro del genuino institucionalismo politológico, mientras que las dos últimas han encontrado también desarrollo en los ámbitos de la Sociología, los estudios de Relaciones Internacionales y el Derecho Internacional Público. Vamos a dar las claves interpretativas de cada uno de estos cuatro enfoques primarios.

\section{El nuevo institucionalismo}

El institucionalismo normativo es el modelo matriz de esta recuperación moderna de la perspectiva después del periodo behaviourista. Frente al individualismo del conductismo, el institucionalismo normativo considera que, aun siendo importantes los individuos, es más determinante en su modo de actuar la pertenencia a una serie de instituciones políticas. Esa pertenencia no tiene naturaleza obligatoria o coercitiva, sino que es normativa, esto es, los individuos se sienten inducidos a actuar no obligados por las normas de la institución, sino empujados e imbuidos por los valores que estas representan. De tal manera que son las instituciones las que de alguna manera influyen y moldean el comportamiento de los individuos.
Esta distinción la plasman March y Olsen, diferenciando procesos políticos aditivos e integradores. En los primeros, la acción de los individuos ante las organizaciones está en función del interés particular. Por el contrario, en los procesos integradores, los individuos participan en las organizaciones y en las instituciones no por cálculos racionales de maximización del interés, sino porque se sienten comprometidos con la legitimidad y los valores que la institución transmite (March y Olsen, 1989).

Por ello, desde una perspectiva sociológica, también se ha denominado a esta corriente institucionalismo mítico, respondiendo con este calificativo a la idea de que los individuos no actúan como partes desgajadas de un todo, sino respondiendo de manera conjunta, socializados, por valores o relatos de naturaleza subjetiva, como son los propios mitos. Por ello los antecedentes de este enfoque pueden cifrarse en autores de gran peso en la tradición sociológica, como Max Weber y Émile Durkheim. Para el sociólogo francés los símbolos desempeñan una función primaria en la socialización de los individuos. Más recientemente, en esta línea, Philip Selznick diferenció entre organizaciones como instituciones normativas o como instituciones que expresan la acción racional (Selznick, 1949).

Los inspiradores de este enfoque recuperador del institucionismo son los mencionados March y Olsen, y se encuentra desarrollado en algunas de sus publicaciones más importantes (March y Olsen, 1984). Para ellos, y desde este enfoque, una institución sería un "conjunto de reglas y valores, normativos y no cognitivos respecto de la manera en que influyen sobre los miembros institucionales, como también el conjunto de rutinas que se desarrollan para poner en ejecución e imponer esos valores" (Peters, 2003).

El segundo enfoque es el institucionalismo de la elección racional - Racional Choice Institutionalism-. Si las teorías de la elección racional basan su análisis en el estudio de la conducta individual que desemboca en la adopción de una elección, de una opción, la versión institucionalista de este gran enfoque behaviourista colocará a las instituciones políticas en el punto intermedio entre la deliberación racional del individuo y la consecuencia final de tal opción individual convertida en acción social. Como señala Guy Peters, "las instituciones son interpretadas como conjuntos de reglas e incentivos que fijan las condiciones para la racionalidad restringida y establecen un «espacio político» dentro del cual pueden funcionar muchos actores independientes (...) las instituciones como mecanismo para canalizar y constreñir la conducta individual" (Peters, 2003). 
Las teorías de la Racional Choice se han desarrollado con profusión y en diferentes orientaciones. Es por ello que Guy Peters, a la hora de analizar el institucionalismo que entronca con estas teorías, lo hace diferenciando tres grandes ámbitos. 1) El que concibe las instituciones políticas como conjunto de reglas o como reglamentaciones expresadas en un lenguaje compartido por la comunidad, que establecen: cómo y quién toma las decisiones, cómo se maneja la información necesaria para adoptarlas y cómo se integran las decisiones individuales en las colectivas (Kiser y Ostrom, 1982). Desde esta perspectiva, Elinor Ostrom identifica las instituciones con un conjunto de reglas que los miembros de una organización o una institución aceptan cumplir en espera de obtener determinados beneficios (Ostrom, 1990). Podríamos decir que los individuos racionalizan su toma de decisiones en el marco previsto por la reglamentaciones que regulan la vida colectiva de las organizaciones.

Una variedad de esta interpretación de las instituciones como conjunto de reglamentaciones que ordenan la acción colectiva es la que se extrae de autores como Kenneth Arrow o James Buchanan. Las instituciones reglamentan de manera anticipada cómo se toman las decisiones; y si hipotéticamente surge algún problema, entonces las reglas detallan qué actor dominante o de qué manera se ha de adoptar la opción final. Esta es la idea de Arrow, y en la misma línea está lo plasmando por Buchanan y Tullock en The Calculus of Consent (Arrow, 1962). Una segunda variedad muy criticada es la que observa a los individuos dentro de las instituciones como maximizadotes del beneficio personal; y para ello utilizan las instituciones y su propia estructura organizativa. Trabajos destacados en esta línea son los de William Niskanen, Anthony Downs, Fiorina y Krehbiel, entre otros (Aldine-Atherton, 1971; Downs, 1967; Elgar, 1994; Fiorina, 1996; Krewhbiel, 1991; Niskanen, 1971).

2) El relativo a la perspectiva de los modelos articulados sobre la relación jefe-agente, en la que un dirigente en el seno de una organización no actúa racionalmente para maximizar su beneficio propio - sería el modelo anterior-, sino que su objetivo de interacción institucional es obtener más dotaciones presupuestarias, ampliar el aparato burocrático a su disposición o aumentar su "espacio político" de actuación. Esta perspectiva queda señalada en diferentes trabajos de los que destacamos algunos recientes editados en los noventa, así los de Wildavsky y Blais y Dion (Blais y Dion, 1992).

3) El que fusiona el institucionalismo con las teorías de la elección racional en su variante más empírica de la teoría del juego. En este escenario planteamos un conjunto de juegos entre dos grandes tipos de actores, unos interactuando sobre otros, generalmente legisladores ante burócratas. La característica diferencial ahora es que en el diseño de este juego se prevé el acatamiento de normas y reglamentos institucionalizados. Ya no es un juego abierto y desentendido, sino estructurado institucionalmente. Por ello entran en funcionamiento factores como la desobediencia o la deserción. El interés se centra ahora en el diseño de estrategias de máxima cooperación que castiguen lo menos posible y generen recompensas. En otros términos, el acatamiento y el cumplimiento de las reglas institucionales generan resultados compensados para los actores que juegan con múltiples variables, siempre con el horizonte final de maximizar réditos. Autores interesantes que han trabajado en esta dirección durante los noventa son F. W. Scharpf y R. L. Calvert (Scharpf, 1995).

El tercer enfoque es el institucionalismo histórico-Historical Institutionalism-. Desde esta perspectiva, se incide en los efectos temporales de las estructuras institucionales. Cuando se forma una institución política genera -a partir de sus normas constitutivas y de su estructura primigenia- un impacto cuyos efectos se prolongan en el tiempo y son difíciles de cambiar; se toma un rumbo cuya inercia es difícil de alterar y que en la terminología de este modelo de institucionalismo se denomina dependencia del rumbo. Esta huella originaria no se reproduce en el tiempo histórico de manera mecánica o directa, sino que puede sufrir procesos adaptativos y evolutivos más complejos.

\section{Reflexiones}

Entre los autores que han reflexionado en esta dirección se encuentran Theda Skocpol, S. Krasner, Peter Hall y Steinmo, Thelen y Longstreth (Krasner, 1984; Skocpol, 1992; Steinmo, Thelen y Longstreth, 1992). La esfera socioeconómica es la preferida por estos autores para enmarcar las reflexiones sobre la dependencia del rumbo institucional, especialmente en el sector público. Para estos autores una institución es una idea más vaga y amplia que lo planteado en otras perspectivas y hace referencia a reglamentaciones, procedimientos y estructuras gubernamentales. Por ejemplo, las leyes electorales pueden configurar instituciones con un fuerte impacto histórico en la medida que condicionan a lo largo del tiempo muchas de las políticas de los partidos y los agentes sociales.

Un elemento destacado como básico para entender el institucionalismo histórico es el influjo de las ideas. En este aspecto, el autor de referencia es Peter Hall, cuyos estudios sobre el impacto de las ideas económicas - las corrientes keynesiana o monetarista - en el mundo de la política ha alcanzado gran relevancia (Hall, 1986; Hall 
y Taylor, 1996). Existen determinadas organizaciones, más allá del estricto ámbito económico, fuertemente determinadas en su estructura y comportamiento por las ideas motrices fundacionales, por ejemplo, las instituciones y las organizaciones ecologías y ambientalistas, o las instituciones médicas y sanitarias.

El cuarto enfoque es institucionalismo empírico -Empirical Institutionalism-. Los autores de esta corriente aparecen en sus formulaciones muy próximos, junto con los normativos, a los primeros institucionalistas y utilizan categorías muy clásicas o convencionales (Peters, ... ). En la medida que las instituciones gubernamentales determinan las políticas y las decisiones de la vida colectivas, los empíricos tratan de cifrar el grado de influencia y el impacto de las fórmulas institucionales en la cotidianeidad política. ¿Qué forma política institucional es la que mejor se adapta y mejores resultados ofrece a una sociedad determinada? Esta pregunta recuerda de manera muy próxima a las formulaciones clásicas del institucionalismo que analizaban los regímenes políticos y sus tipologías. En determinado momento la respuesta a esta cuestión puede resultar difícil por la falta de perspectiva histórica que apoye o refute el funcionamiento de las instituciones, pero los empíricos sustituyen esta falta de recorrido temporal con apoyos teóricos.

Varias son derivaciones relacionadas con el tipo de parámetros de comparación sobre los que los autores trabajan. La primera es la diferencia entre las instituciones del gobierno presidencialista y el parlamentario: cuáles son más estables -estabilidad en clave de exitosa permanencia en el tiempo-; qué capacidad legislativa tienen -en qué contexto la elaboración de leyes resulta más fluida o más difícil y con más obstáculos que salvar-;qué tipo de políticas son capaces de implantar -intervenciones simples o más complejas y radicales - (Weaber y Rockman, 1993). En definitiva detrás de todos estos interrogantes estaría la pregunta de Arendt Lijphart sobre qué tipo de gobiernos son capaces de gobernar más eficazmente, resumiendo en la idea de eficaz varios de los parámetros antes presentados (Lijphart, 1984).

Una segunda versión del institucionalismo empírico es la que gira en torno a los debates sobre los denominados gobierno dividid, instituciones separadas o cohabitación, esto es, aquellas situaciones institucionales en las que los partidos o las personas que lideran y gobiernan en las instituciones son de signo ideológico contrario o al menos diferente. Esto sucede cuando la mayoría de las cámaras y la presidencia en los Estados Unidos no coinciden, o cuando el presidente de la República y el jefe del gobierno en Francia son de signo político diferente o contrario. ¿En qué situación se toman decisiones políticas con mayor eficacia? (Fiorina y Sudnquist, 1988; Jones, 1995; Duverger, 1991).

Una última versión del institucionalismo empírico investiga sobre la institucionalización de los parlamentos de los cuerpos legislativos. El origen de esta perspectiva se encuentra en el análisis de Nelson Polsby -realizado en 1968- sobre la institucionalización de la Cámara de Representantes de los Estados Unidos (Polsby, 1968). Durante la década de los ochenta y los noventa han florecido múltiples estudios sobre el funcionamiento institucional de las cámaras legislativas, preguntándose por su grado de institucionalización: qué nivel de profesionalización tienen sus miembros; cuál es su régimen de dedicación; cuáles son -si existen - los valores compartidos entre los miembros de las cámaras, independientemente de sus filiaciones partidistas e ideológicas.

\section{Referencias}

Almond, G.A. (1988). "The Return to the State", American Political Science Review, n. ${ }^{\circ}$ 82, pp. 853 a 874. P. B. Evans, D. Rueschmeyer y T. Skcopol, Bringing the State Back In, Cambridge, Cambridge University Press, 1985.

Arrow, K. (1974). Social Choice and Individual Values, Nueva York, Wiley [Traducción española: Elección social y valores individuales, Madrid, Ministerio de Hacienda, 1974].

Blais, A. y Dion, S. (1991). The Budget-Maximizing Bureaucrat: Appraisals and Evidence, Pittsburgh, Pittsburgh University Press.

Buchanan, J.M. y Tullock, G. (1962). The Calculus of Consent: Logical Foundation of Constitutional Democracy, Ann Arbor, University of Michigan Press.

Calvert, R.L. (1995). "The Rational Choice Theory of Institutions: Implications for Design", en D. Weimer (Comp.), Institutional Design, Dordrecht, Kluwer.

Elgar, E., Downs, E. (1994). Inside Bureaucracy, Boston, Little Brown, 1967

Fiorina, M.P. (1996). Divided Government, Boston, Allyn and Bacon. (2. ${ }^{\text {e ed.). }}$.

Fiorina, M.P. en J.L. (1988). Sudnquist, "Needed: A Political Theory of the New Era Coalition Government in the United States", Political Science Quaterly, n. ${ }^{\circ} 103, \mathrm{pp}$. 613 a 635.

Hall, P. y Taylor, R. (1996). "Political Science and the Three New Institutionalism", Political Studies, 44 (4), pp. 936 a 957.

Hall, O. (1996). Governing the Economy: The Politics of State Intervention in Britain and France, Nueva York, Oxford 
University Press, 1986; The Power of Economic Ideas, Princeton, Princeton University Press, 1989; Peter Hall y C.R. Taylor, "Political Science and the Three New Institutionalism", Political Studies, n. ${ }^{\circ} 44$, pp. 952 a 973.

Jones, C.O. (1987). Separate but Equal Branches: Congress and the Presidency, Chatham, Chatham House, 1995; Maurice Duverger, Les regimes semi-présidentiels, París, Presses Universitaires de France.

Kiser, L. y Ostrom, E. (1982). "The Three Worlds of Action: A Metatheoretical Synthesis of Institutional Approaches", en Elinor Ostrom (Comp.), Strategies of Political Inquiry, Beverly Hills, Sage, p. 179. Cit., por: B. Guy Peters, op. cit., p. 85.

Krehbiel, K. (1991). Information and Legislative Organization, Ann Arbor, University of Michigan Press.

Krasner, S. (1984). "Approaches to the State: Alternative Conceptions and Historical Dynamics", Comparative Politics, n. ${ }^{\circ} 16$, pp. 226 a 246.

Lijphart, A. (1984). Democracies: Patterns of Majoritarian and Consensus Government in Twenty-one Countries, New Haven, Yale University Press.

"Democracies: Forms, Performance and Constitutional Engineering", European Journal of Political Research, n. ${ }^{\circ} 25,1994$, pp. 1 a 17.

Lowndes, V. (2002). "Institutionalism", en David Marsh y G. Stoker (Eds.), Theory and Methods in Political Science, Basingstoke, Palgrave, pp. 90 a 108.

Marsh, J. y Olsen, J. (1984). "The New Institutionalism: Organizational Factors in Political Life", American Political Science Review, n. ${ }^{\circ}$ 78, pp. 738 a 749.

March, J. y Olsen, J. P. (1989) Rediscovering Institutions, Nueva York, Free Press, pp. 118 y ss. Cit. por. B. Guy Peters, op. cit., pp. 47 y 48.

Marsh, D. y Savigny, H.,(2004) "Political Science as a Broad Church: The Search for a Pluralist Discipline", Politics, vol. 24, n. ${ }^{\circ}$, September, p. 157.

Ostrom, E. (1994) Governing the Commons: The Evolution of Institutions of Collective Action, Cambridge,
Cambridge University Press, 1990; Elinor Ostrom, R. Gardner y J. Walter, Rules, Games, and CommonPool Resources, Ann Arbor, University of Michigan Press.

Niskanen, W. (1971) Bureaucracy and Representative Government, Chicago, Aldine-Atherton.

Peters, G. B., (2003) Institutional Theory in Political Science, Londres, Pinter, 1999 [Traducción española: El nuevo institucionalismo, Barcelona, Gedisa, p. 44.]

Pierce, R. (1991). "The Executive Divided Against Itself: Cohabitation in France 1986-1988", Governance, n. ${ }^{\circ}$ 4, pp. 270 a 294.

Polsby, N. (1968). "The Institutionalization of the U.S. House of Representatives", American Political Science Review, n. 62 , 1968, pp. 144 a 168.

Scharpf, F.W. (1997). Games Real Actors Play: Actorcentered Institutionalism in Policy Research, Boulder, Westview Press.

Selznick, P., (1949) TVA and the Grass Roots, Berkeley, California University Press, Cit for B. Guy Peters, $p$. 47.

Sobre la cuestión de la complejidad en las políticas implementadas: R.K. Weaver y B.A. Rockman, Do Institutions Matter?: Government Capabilities in the United States and Abroad, Washington, Brookings Institution, 1993.

"Sovereignty: An Institutional Perspective", Comparative Political Studies, n. ${ }^{\circ} 21,1988$, pp. 66 a 94. S. Steinmo, K. Thelen y F. Longstreth, Structuring Politics: Historical Institutionalism in Comparative Analysis, Cambridge, Cambridge University Press, 1992.

Theda Skocpol, Protecting Soldiers and Mothers: The Political Origins of Social Policy in the United States, Cambridge, Cambridge University Press, 1992 [Traducción española: Los orígenes de la política social en los Estados Unidos, Madrid, Ministerio de Trabajo y Asuntos Sociales, 1996].

Wildavsky, A. (1992). The New Politics of the Budgetary Process, Nueva York, Harper Collins (2. ${ }^{a}$ ed). 